\title{
ON THE CALMNESS OF A CLASS OF MULTIFUNCTIONS*
}

\author{
RENÉ HENRION ${ }^{\dagger},{\text { ABDERRAHIM JOURANI }{ }^{\ddagger}, \text { AND JIŘI OUTRATA }}^{\S}$ \\ Dedicated to Jochem Zowe on the occasion of his sixtieth birthday
}

\begin{abstract}
The paper deals with the calmness of a class of multifunctions in finite dimensions. Its first part is devoted to various conditions for calmness, which are derived in terms of coderivatives and subdifferentials. The second part demonstrates the importance of calmness in several areas of nonsmooth analysis. In particular, we focus on nonsmooth calculus and solution stability in mathematical programming and in equilibrium problems. The derived conditions find a number of applications there.
\end{abstract}

Key words. calmness, multifunctions, constraint qualifications, nonsmooth calculus, solution stability, equilibrium problems, weak sharp minima

AMS subject classifications. 90C $31,26 \mathrm{E} 25,49 \mathrm{~J} 52$

PII. S1052623401395553

1. Introduction. The concept of calmness plays a key role in the analysis of Lipschitz properties for multifunctions. It is closely related to issues from optimization theory like nondegenerate multiplier rules (e.g., [10], [2], [4]), existence of error bounds (e.g., [5], [18], [24]), or sensitivity analysis of generalized equations (e.g., [13], [17]). The aim of this paper is to provide subdifferential conditions for ensuring the calmness of constraint systems in finite dimensions and to consider calmness in the context of different applications like nonsmooth calculus or solutions to parametric optimization or equilibrium problems.

We start by recalling some of the prominent Lipschitz properties formulated for multifunctions. Let $M: Y \rightrightarrows X$ be a multifunction between metric spaces. $M$ is said to have the Aubin property around some $(\bar{y}, \bar{x}) \in \mathrm{Gph} M$ (graph of $M$ ) if there exist neighborhoods $\mathcal{V}$ and $\mathcal{U}$ of $\bar{y}$ and $\bar{x}$ as well as some $L>0$ such that

$$
d\left(x, M\left(y_{2}\right)\right) \leq L d\left(y_{1}, y_{2}\right) \quad \forall y_{1}, y_{2} \in \mathcal{V}, \forall x \in M\left(y_{1}\right) \cap \mathcal{U} .
$$

It is well known that $M$ has the Aubin property around $(\bar{y}, \bar{x})$ if and only if its inverse $M^{-1}$ is metrically regular around $(\bar{x}, \bar{y})$ (e.g., [27, Theorem 9.43]). Fixing one of the $y$-parameters as $\bar{y}$ in the definition of the Aubin property yields the calmness of $M$ at $(\bar{y}, \bar{x})$ :

$$
d(x, M(\bar{y})) \leq L d(y, \bar{y}) \quad \forall y \in \mathcal{V}, \forall x \in M(y) \cap \mathcal{U} .
$$

Obviously, the Aubin property implies calmness, whereas the converse is not true (e.g., $M(y)=\left\{x \mid x^{2} \geq y\right\}$ at $(0,0)$ ). If one may choose $\mathcal{U}=X$ in this last definition, then the calmness becomes the slightly stronger local upper Lipschitz property introduced in $[25]$.

\footnotetext{
${ }^{*}$ Received by the editors September 25, 2001; accepted for publication January 17, 2002; published electronically October 1, 2002.

http://www.siam.org/journals/siopt/13-2/39555.html

${ }^{\dagger}$ Weierstrass Institute Berlin, 10117 Berlin, Germany (henrion@wias-berlin.de).

${ }^{\ddagger}$ Département de Mathématiques, Université de Bourgogne, 21078 Dijon, France (Abderrahim. Jourani@u-bourgogne.fr).

$\S$ Institute of Information Theory and Automation, 18208 Prague, Czech Republic (outrata@ utia.cas.cz). The research of this author was supported by grant 201/00/0080 of the Grant Agency of the Czech Republic.
} 
A restricted version of calmness, namely, calmness on selections, has been studied in the context of sensitivity analysis for generalized equations [13], [15], [6]. Here it is required that $\mathcal{U} \cap M(\bar{y})=\{\bar{x}\}$ in the general definition of calmness, i.e., $\bar{x}$ is isolated in $M(\bar{y})$. Such an assumption is relevant, for instance, when analyzing solutions to nonlinear optimization problems. Moreover, one may even further restrict calmness by combining it with the local uniqueness of $M$ at $(\bar{y}, \bar{x})$. Then, locally around $(\bar{y}, \bar{x})$, $M$ is just a usual function satisfying the condition

$$
d(M(y), M(\bar{y})) \leq L d(y, \bar{y}) .
$$

This situation was studied, for instance, in [16].

For the purpose of verifying the Lipschitz properties of multifunctions, it is useful to have suitable criteria from nonsmooth calculus. Such criteria have proven to be particularly efficient in finite dimensions. For instance, $X$ and $Y$ being finitedimensional, the Aubin property of a closed graph multifunction $M$ is equivalent to the condition (see [21])

$$
D^{*} M(\bar{y}, \bar{x})(0)=\{0\} .
$$

Here, $D^{*}$ refers to Mordukhovich's coderivative (see section 2). This is a dual criterion that relies on a normal cone construction to the graph of $M$. Similar dual conditions were given in [20, Theorem 5.4] for a property related to but different from calmness.

An equivalent primal criterion for the Aubin property can be formulated in terms of the contingent derivative $D$, based on the contingent cone to Gph $M$ (see [1, Theorem 4, p. 431] for sufficiency in arbitrary Banach spaces and, e.g., [7, Corollary 1.19] for necessity in the case of finite-dimensional $X$ ):

$$
\exists \alpha>0, \beta>0: B(0,1) \subseteq[D M(y, x)]^{-1}(B(0, \alpha)) \quad \forall(y, x) \in \operatorname{Gph} M \cap B((\bar{y}, \bar{x}), \beta) .
$$

Here, $B(z, r)$ refers to the closed ball around $z$ with radius $r$. As far as corresponding criteria for calmness are concerned, the following primal condition was found to be sufficient in [13, Proposition 2.1] and necessary in [15, Proposition 4.1] for calmness on selections in finite dimensions:

$$
D M(\bar{y}, \bar{x})(0)=\{0\} .
$$

Note that this condition immediately enforces the isolatedness of $\bar{x}$ in $M(\bar{y})$ because a sequence $x_{n} \rightarrow \bar{x}, x_{n} \in M(\bar{y}), x_{n} \neq \bar{x}$ would generate a nontrivial tangent vector $(0, \xi)$ to $\operatorname{Gph} M$ at $(\bar{y}, \bar{x})$, whence a contradiction $0 \neq \xi \in D M(\bar{y}, \bar{x})(0)$ to the above condition.

Calmness in the broader sense introduced above is closely related to the regularity concept of Ioffe studied in [10], [11], even in a Banach space setting. In fact, in [11] a sufficient condition for calmness has been derived for multifunctions of the type

$$
M(y)=\{x \in C \mid g(x)=y\}
$$

in terms of Clarke's subdifferential. Another sufficient condition for calmness in the broader sense was given in [8] for multifunctions of the type

$$
M(y)=\{x \in C \mid g(x)+y \in D\},
$$

where $g: \mathbb{R}^{k} \rightarrow \mathbb{R}^{m}$ is locally Lipschitz and $C \subseteq \mathbb{R}^{k}, D \subseteq \mathbb{R}^{m}$ are closed. It was shown there that under mild assumptions the calmness of $M$ is implied by the condition

$$
\bigcup_{y^{*} \in N_{D}(g(\bar{x})) \backslash\{0\}} D^{*} g(\bar{x})\left(y^{*}\right) \cap\left(-\operatorname{bd} N_{C}(\bar{x})\right)=\emptyset,
$$


where "bd" refers to the topological boundary. Recalling that the criterion (1.1) for the Aubin property reduces in the special setting of (1.4) to the sufficient condition

$$
\bigcup_{y^{*} \in N_{D}(g(\bar{x})) \backslash\{0\}} D^{*} g(\bar{x})\left(y^{*}\right) \cap\left(-N_{C}(\bar{x})\right)=\emptyset,
$$

the reduction from the stronger Aubin to the weaker calmness property in (1.4) is reflected by a transition from a normal cone to its boundary in the criteria (1.5) and (1.6), respectively. Under some additional regularity assumptions, one may even pass to the boundary in the left part of (1.6). In [9], attempts were made to extend these ideas to the infinite-dimensional case, but it seems to be difficult to pass beyond convex or differentiable structures in this framework. For instance, if $f$ is a locally Lipschitz function, regular in the sense of Clarke and satisfying $f(0)=0$, then the condition $0 \notin$ bd $\partial f(0)$ guarantees calmness of the parametric inequality $f(x) \leq y$ at $(0,0)$ as long as either $f$ is defined on a finite-dimensional space [8, Theorem 4.2] or $f$ is convex on a Banach space [9, Corollary 3.4]. In contrast, one may construct a locally Lipschitz $f$ defined on the sequence space $l^{1}$ which is Clarke regular and nonconvex such that the mentioned condition is satisfied but calmness fails to hold.

The paper is organized as follows: first, subdifferential criteria for calmness in finite dimensions are developed which extend those given in [8]. In particular, the multifunction $M$ in (1.4) gets the more general form $M(y)=S(y) \cap C$, with a purely parametric contribution by $S$ and a nonparametric contribution by $C$. In a second part, calmness (as a condition by itself or implied by the previously derived subdifferential criteria) is studied in several applications like nonsmooth calculus, stability of solutions to nonsmooth optimization problems, and equilibrium problems.

2. Notation and basic concepts. In the following, we denote by $\partial f(x)$ and $N_{C}(x)$, respectively, the subdifferential of a function $f$ at some $x$ and the normal cone to some closed set $C$ at some $x \in C$, both in the sense of Mordukhovich. In contrast, $T_{C}(x)$ refers to the contingent cone. Note that if $f$ is regular in the sense of Clarke, then $\partial f(x)$ coincides with Clarke's subdifferential. Similarly, if $C$ is a regular set at $x$, then $T_{C}(x)$ and $N_{C}(x)$ coincide with Clarke's tangent and normal cone, respectively. In that case it also holds true that each one of these cones is the (negative) polar cone of the other. With a multifunction $Z: \mathbb{R}^{p} \rightrightarrows \mathbb{R}^{k}$ and some $(\bar{u}, \bar{v}) \in \mathrm{Gph} Z$ we associate Mordukhovich's coderivative $D^{*} Z(\bar{u}, \bar{v}): \mathbb{R}^{k} \rightrightarrows \mathbb{R}^{p}$ defined by

$$
D^{*} Z(\bar{u}, \bar{v})\left(v^{*}\right)=\left\{u^{*} \in \mathbb{R}^{p} \mid\left(u^{*},-v^{*}\right) \in N_{\mathrm{Gph} Z}(\bar{u}, \bar{v})\right\} .
$$

If $Z$ is single-valued, we simply write $D^{*} Z(\bar{u})$ instead of $D^{*} Z(\bar{u}, Z(\bar{u}))$. For singlevalued, locally Lipschitz mappings $Z$ it holds that

$$
D^{*} Z(\bar{u})\left(v^{*}\right)=\partial\left\langle v^{*}, Z\right\rangle(\bar{u}) .
$$

For a detailed presentation of these concepts, we refer to [20], [22], [27] and [4].

By $B(x, r), \mathbb{B}$, and $\mathbb{S}$ we shall denote a closed ball centered at $x$ with radius $r$, the closed unit ball, and the unit sphere in corresponding spaces, respectively. By $d(x, C)$ we denote the point-to-set distance between $x$ and $C$ induced by a corresponding norm on $\mathbb{R}^{n}$, whereas $d_{C}^{e}(x)$ represents the particular case of the Euclidean distance function.

A basic concept which we shall use in the derivation of subdifferential criteria for calmness is semismoothness as introduced in [19]. 
Definition 2.1. A function $\psi: \mathbb{R}^{k} \rightarrow \mathbb{R}$ is called semismooth at $\bar{x} \in \mathbb{R}^{k}$ if it is locally Lipschitz around $\bar{x}$ and the following property holds true: for each $d \in \mathbb{R}^{k}$ and for any sequences $t_{n} \downarrow 0, d_{n} \rightarrow d, x_{n}^{*} \in \partial \psi\left(\bar{x}+t_{n} d_{n}\right)$, the limit $\lim _{n \rightarrow \infty}\left\langle x_{n}^{*}, d\right\rangle$ exists.

It has to be noted that in the original definition of [19], the corresponding property was required for Clarke's subdifferential of $\psi$. However, exploiting the well-known fact that Clarke's subdifferential is the closed convex hull of Mordukhovich's, it easily follows that both definitions of semismoothness are equivalent. As a consequence of Definition 2.1, a semismooth function $\psi$ has a conventional directional derivative $\psi^{\prime}(\bar{x} ; h)$ at $\bar{x}$ in direction $d$ which coincides with the common limit in Definition 2.1.

As with Clarke regularity, semismoothness of functions can be carried over to sets.

Definition 2.2. A set $A \subseteq \mathbb{R}^{k}$ is called semismooth at $\bar{x} \in \operatorname{cl} A$ if for any sequence $x_{n} \rightarrow \bar{x}$ with $x_{n} \in A$ and $\left\|x_{n}-\bar{x}\right\|^{-1}\left(x_{n}-\bar{x}\right) \rightarrow d$ it holds that $\left\langle x_{n}^{*}, d\right\rangle \rightarrow 0$ for all selections of subgradients $x_{n}^{*} \in \partial d_{A}^{e}\left(x_{n}\right)$.

If $A$ is closed and $d_{A}^{e}$ is semismooth in the sense of Definition 2.1, then $A$ is semismooth in the sense of Definition 2.2 (see [8, Proposition 2.4]).

3. Subdifferential characterization of calmness. We start with an auxiliary result which is crucial for passing to the boundary of the normal cone in (1.5) and in the corresponding generalization we have in mind.

Proposition 3.1. Let $C \subseteq \mathbb{R}^{k}$ be regular (in the sense of Clarke) and semismooth at $\bar{x} \in C$. Consider a sequence $x_{n} \rightarrow \bar{x}$ such that $x_{n} \in C$ and $\left\|x_{n}-\bar{x}\right\|^{-1}\left(x_{n}-\bar{x}\right) \rightarrow h$ with $\|h\|=1$. Then each accumulation point $x^{*}$ of a sequence $x_{n}^{*} \in \partial d_{C}^{e}\left(x_{n}\right)$ belongs to bd $N_{C}(\bar{x})$.

Proof. By virtue of the semismoothness of $C$ at $\bar{x}$, one has $\left\langle x^{*}, h\right\rangle=0$. From $\partial d_{C}^{e}\left(x_{n}\right) \subseteq N_{C}\left(x_{n}\right)$ and from the closedness of the mapping $N_{C}(\cdot)$, it follows that $x^{*} \in N_{C}(\bar{x})$. By construction, $h \in T_{C}(\bar{x})$; hence regularity of $C$ at $\bar{x}$ implies that $\left\langle y^{*}, h\right\rangle \leq 0$ for all $y^{*} \in N_{C}(\bar{x})$. For arbitrary $\varepsilon>0$, one has $\left\langle x^{*}+\varepsilon h, h\right\rangle=\varepsilon>0$, whence $x^{*}+\varepsilon h \notin N_{C}(\bar{x})$. Along with $x^{*} \in N_{C}(\bar{x})$, this means that $x^{*} \in \operatorname{bd} N_{C}(\bar{x})$.

Consider now a multifunction $M: \mathbb{R}^{p} \rightrightarrows \mathbb{R}^{k}$ defined as the intersection $M(y)=$ $S(y) \cap C$, where $S: \mathbb{R}^{p} \rightrightarrows \mathbb{R}^{k}$ is a multifunction with closed graph and $C \subseteq \mathbb{R}^{k}$ is closed. As a consequence, $M$ has closed graph as well. The following theorem is the main result of this section.

Theorem 3.2. Consider some $(\bar{y}, \bar{x}) \in \mathrm{Gph} M$. Assume that $C$ is regular and semismooth at $\bar{x}$. If for all $y^{*} \in \mathbb{R}^{p}$ it holds that

$$
D^{*} S^{-1}(\bar{x}, \bar{y})\left(y^{*}\right) \cap-\operatorname{bd} N_{C}(\bar{x})=\left\{\begin{array}{cl}
\emptyset & \text { or } \\
\{0\} & \text { if } y^{*}=0,
\end{array}\right.
$$

then $M$ is calm at $(\bar{y}, \bar{x})$. (Note that the case $D^{*} S^{-1}(\bar{x}, \bar{y})(0) \cap-\operatorname{bd} N_{C}(\bar{x})=\emptyset$ is formally included in (3.1).)

Proof. Assume by contradiction that $M$ is not calm at $(\bar{y}, \bar{x})$. By definition, there exist sequences $x_{n} \rightarrow \bar{x}, y_{n} \rightarrow \bar{y}, x_{n} \in M\left(y_{n}\right)$ such that $d\left(x_{n}, M(\bar{y})\right)>n\left\|y_{n}-\bar{y}\right\|$. Now, set $h(y, x):=\|y-\bar{y}\|$ so that each pair $\left(y_{n}, x_{n}\right)$ is an $\varepsilon$-minimizer of $h(y, x)$ over Gph $M$ with $\varepsilon=\left\|y_{n}-\bar{y}\right\|$. The application of the Ekeland variational principle with $\varepsilon$ and $\lambda:=n \varepsilon$ to the minimization of $h$ over Gph $M$ yields for each $n$ the existence of a pair $\left(\tilde{y}_{n}, \tilde{x}_{n}\right) \in \operatorname{Gph} M$ such that for all $(y, x) \in \operatorname{Gph} M$

$$
\left\|\left(\tilde{y}_{n}, \tilde{x}_{n}\right)-\left(y_{n}, x_{n}\right)\right\| \leq n\left\|y_{n}-\bar{y}\right\|
$$




$$
\left\|\tilde{y}_{n}-\bar{y}\right\| \leq\|y-\bar{y}\|+n^{-1}\left\|(y, x)-\left(\tilde{y}_{n}, \tilde{x}_{n}\right)\right\| .
$$

From (3.2) we infer that

$\left\|\left(\tilde{y}_{n}, \tilde{x}_{n}\right)-(\bar{y}, \bar{x})\right\| \leq n\left\|y_{n}-\bar{y}\right\|+\left\|\left(y_{n}, x_{n}\right)-(\bar{y}, \bar{x})\right\|<d\left(x_{n}, M(\bar{y})\right)+\left\|\left(y_{n}, x_{n}\right)-(\bar{y}, \bar{x})\right\|$

so that $\left(\tilde{y}_{n}, \tilde{x}_{n}\right) \rightarrow(\bar{y}, \bar{x})$. Furthermore, $\tilde{y}_{n} \neq \bar{y}$ and $\tilde{x}_{n} \neq \bar{x}$, because otherwise $\tilde{x}_{n}$ $\in M(\bar{y})$, whence the contradiction

$$
n\left\|y_{n}-\bar{y}\right\|<d\left(x_{n}, M(\bar{y})\right) \leq\left\|x_{n}-\tilde{x}_{n}\right\| \leq n\left\|y_{n}-\bar{y}\right\|,
$$

using (3.2). Now, (3.3) means that $\left(\tilde{y}_{n}, \tilde{x}_{n}\right)$ is a (global) solution of the problem

$$
\min \left\{\|y-\bar{y}\|+n^{-1}\left\|(y, x)-\left(\tilde{y}_{n}, \tilde{x}_{n}\right)\right\| \mid(y, x) \in \operatorname{Gph} M\right\} .
$$

Since Gph $M=\operatorname{Gph} S \cap\left(\mathbb{R}^{p} \times C\right)$, it follows that exactly one of the following cases occurs (with $\mathbb{S}$ denoting the unit sphere):

$$
\begin{gathered}
\{0\}=N_{\mathrm{Gph} S}\left(\tilde{y}_{n}, \tilde{x}_{n}\right) \cap\left[\{0\} \times\left(-N_{C}\left(\tilde{x}_{n}\right)\right)\right], \\
\exists \xi_{n} \in \mathbb{S} \cap N_{\mathrm{Gph} S}\left(\tilde{y}_{n}, \tilde{x}_{n}\right) \cap\left[\{0\} \times\left(-N_{C}\left(\tilde{x}_{n}\right)\right)\right] .
\end{gathered}
$$

At least one of these two cases must apply for infinitely many $n$. Suppose first that this is true for (3.5). Without loss of generality, we assume that (3.5) is valid for all $n$. Then (see [27, Theorem 6.4.2])

$$
N_{\mathrm{Gph} M}\left(\tilde{y}_{n}, \tilde{x}_{n}\right) \subseteq N_{\mathrm{Gph} S}\left(\tilde{y}_{n}, \tilde{x}_{n}\right)+\left[\{0\} \times N_{C}\left(\tilde{x}_{n}\right)\right] .
$$

Application of the necessary optimality conditions to the solution $\left(\tilde{y}_{n}, \tilde{x}_{n}\right)$ of the constrained problem (3.4) then yields

$$
0 \in\left[\mathbb{S}_{y} \times\{0\}\right]+n^{-1} \mathbb{B}+N_{\mathrm{Gph} S}\left(\tilde{y}_{n}, \tilde{x}_{n}\right)+\left[\{0\} \times N_{C}\left(\tilde{x}_{n}\right)\right],
$$

where $\mathbb{S}_{y}$ refers to the unit sphere in $\mathbb{R}^{p}$ (and occurs due to $\tilde{y}_{n} \neq \bar{y}$ ) and $\mathbb{B}$ is the unit ball in $\mathbb{R}^{p} \times \mathbb{R}^{k}$. Without loss of generality, $\mathbb{B}$ is taken with respect to the maximum norm; hence $\mathbb{B}=\mathbb{B}_{y} \times \mathbb{B}_{x}$. Accordingly, there exist $\left(y_{n}^{*}, z_{n}^{*}\right) \in N_{\mathrm{Gph} S}\left(\tilde{y}_{n}, \tilde{x}_{n}\right)$ and $x_{n}^{*} \in-N_{C}\left(\tilde{x}_{n}\right)$ such that

$$
0 \in \mathbb{S}_{y}+n^{-1} \mathbb{B}_{y}+y_{n}^{*} \quad \text { and } \quad\left\|x_{n}^{*}-z_{n}^{*}\right\| \leq n^{-1}
$$

By the boundedness of $y_{n}^{*}$ we may assume that $y_{n}^{*} \rightarrow y^{*} \in \mathbb{S}_{y}$.

If $\left\{x_{n}^{*}\right\}$ is unbounded, then for $\hat{x}_{n}^{*}:=\left\|x_{n}^{*}\right\|^{-1} x_{n}^{*}$ we may assume that $\hat{x}_{n}^{*} \rightarrow x^{*}$ for some $x^{*} \in \mathbb{S}_{x}$. Furthermore, $\hat{x}_{n}^{*} \in-N_{C}\left(\tilde{x}_{n}\right)$ and

$$
d_{N_{\mathrm{Gph} S}\left(\tilde{y}_{n}, \tilde{x}_{n}\right)}^{e}\left(\left\|x_{n}^{*}\right\|^{-1} y_{n}^{*}, \hat{x}_{n}^{*}\right) \leq d_{N_{\mathrm{Gph} S}\left(\tilde{y}_{n}, \tilde{x}_{n}\right)}^{e}\left(y_{n}^{*}, x_{n}^{*}\right) \leq \rho\left\|x_{n}^{*}-z_{n}^{*}\right\| \leq \rho n^{-1},
$$

where $d^{e}$ denotes the Euclidean distance function and $\rho>0$ is some modulus relating the Euclidean and maximum norms. Since, without loss of generality, $\left\|x_{n}^{*}\right\|^{-1} y_{n}^{*} \rightarrow 0$, the closedness of the coderivative mapping implies that $x^{*} \in D^{*} S^{-1}(\bar{x}, \bar{y})(0)$. On the other hand, $\hat{x}_{n}^{*} \in-N_{C}\left(\tilde{x}_{n}\right) \cap \mathbb{B}_{x}=-\partial d_{C}^{e}\left(\tilde{x}_{n}\right)$ (see [27, Example 8.5.3]). Recalling that $\tilde{x}_{n} \neq \bar{x}$ and $\tilde{x}_{n} \in C$, Proposition 3.1 provides that $x^{*} \in-\operatorname{bd} N_{C}(\bar{x})$, whence the contradiction $x^{*} \in \mathbb{S}_{x} \cap D^{*} S^{-1}(\bar{x}, \bar{y})(0) \cap-\operatorname{bd} N_{C}(\bar{x})$ with (3.1).

Assuming that $\left\{x_{n}^{*}\right\}$ is bounded instead, one has without loss of generality that

$$
x_{n}^{*} \rightarrow x^{*} \in D^{*} S^{-1}(\bar{x}, \bar{y})\left(y^{*}\right) \cap-N_{C}(\bar{x})
$$


(again by closedness of the coderivative and of the normal cone mapping). Due to $\tilde{x}_{n} \neq \bar{x}$ we have that $T_{C}(\bar{x}) \neq\{0\}$, whence $N_{C}(\bar{x}) \neq \mathbb{R}^{k}$ and $0 \in-\operatorname{bd} N_{C}(\bar{x})$. Now, the case $x^{*}=0$ leads to an immediate contradiction with (3.1) due to $y^{*} \neq 0$. If $x^{*} \neq 0$, then set

$$
\hat{x}_{n}^{*}:=\left\|x_{n}^{*}\right\|^{-1} x_{n}^{*} \rightarrow \hat{x}^{*}:=\left\|x^{*}\right\|^{-1} x^{*},
$$

as before. Invoking Proposition 3.1 in the same way as above, one arrives at $\hat{x}^{*} \in$ $\mathbb{S}_{x} \cap D^{*} S^{-1}(\bar{x}, \bar{y})\left(\left\|x^{*}\right\|^{-1} y^{*}\right) \cap-\operatorname{bd} N_{C}(\bar{x})$ by positive homogeneity of the coderivative mapping. This again is a contradiction with (3.1).

Finally, suppose instead that (3.6) applies for infinitely many $n$. Again, we do not relabel the corresponding subsequence. Then, defining $\xi_{n}=\left(\xi_{n}^{y}, \xi_{n}^{x}\right)$, we may assume without loss of generality that $\xi_{n}=\left(0, \xi_{n}^{x}\right) \rightarrow\left(0, \xi^{x}\right)$, where $\xi_{n}^{x}, \xi^{x} \in \mathbb{S}_{x}$ and, according to (3.6),

$$
\xi_{n}^{x} \in D^{*} S^{-1}\left(\tilde{x}_{n}, \tilde{y}_{n}\right)(0) \cap-N_{C}\left(\tilde{x}_{n}\right)
$$

Consequently, $\xi_{n}^{x} \in-\partial d_{C}^{e}\left(\tilde{x}_{n}\right)$, and we may invoke Proposition 3.1 again to obtain that $\xi^{x} \in-$ bd $N_{C}(\bar{x})$. Summarizing, we arrive at the contradiction

$$
\xi^{x} \in D^{*} S^{-1}(\bar{x}, \bar{y})(0) \cap-\operatorname{bd} N_{C}(\bar{x})
$$

with (3.1).

REMARK 3.3. The assumptions of (Clarke-) regularity and semismoothness for $C$ in Theorem 3.2 are completely independent (see Example 3.5 in [8]). Their joint validity is guaranteed for a sufficiently broad class of closed sets, like convex sets or sets defined by $\mathcal{C}^{1}$-inequalities and satisfying the Mangasarian-Fromovitz constraint qualification (cf. Lemma 3.6 in [8]).

Now, we specialize the above theorem to the parametrized constraint system $x \in C, g(x, y) \in D$, where $g: \mathbb{R}^{k} \times \mathbb{R}^{p} \rightarrow \mathbb{R}^{m}$ is locally Lipschitz and $C \subseteq \mathbb{R}^{k}, D \subseteq \mathbb{R}^{m}$ are closed. We associate with this system the multifunction $M: \mathbb{R}^{p} \rightrightarrows \mathbb{R}^{k}$ defined by

$$
M(y):=\{x \in C \mid g(x, y) \in D\}
$$

Corollary 3.4. In (3.7), let $(\bar{y}, \bar{x}) \in \operatorname{Gph} M$ and $C$ be regular and semismooth at $\bar{x}$. Further, assume the qualification condition

$$
\bigcup_{y^{*} \in N_{D}(g(\bar{x}, \bar{y})) \backslash\{0\}}\left[\partial\left\langle y^{*}, g\right\rangle(\bar{x}, \bar{y})\right]_{x} \cap-\operatorname{bd} N_{C}(\bar{x})=\emptyset,
$$

where []$_{x}$ denotes projection onto the $x$-component. Then $M$ is calm at $(\bar{y}, \bar{x})$.

Proof. The case in which $0 \notin \mathrm{bd} N_{C}(\bar{x})$ is trivial, so assume that $0 \in \mathrm{bd} N_{C}(\bar{x})$. Consider the map $S: \mathbb{R}^{p} \rightrightarrows \mathbb{R}^{k}$ defined by

$$
S(y):=\left\{x \in \mathbb{R}^{k} \mid g(x, y) \in D\right\}
$$

To compute $D^{*} S^{-1}(\bar{x}, \bar{y})$, we invoke a result from [22]. Since $0 \in \operatorname{bd} N_{C}(\bar{x}),(3.8)$ yields in particular the implication

$$
D^{*} g(\bar{x}, \bar{y})\left(v^{*}\right)=0, \quad v^{*} \in N_{D}(g(\bar{x}, \bar{y})) \Longrightarrow v^{*}=0
$$


This is, however, the qualification condition from [22, Theorem 6.10], and so one has for each $v^{*} \in \mathbb{R}^{p}$ the inclusion

$$
\begin{aligned}
D^{*} & S^{-1}(\bar{x}, \bar{y})\left(v^{*}\right) \\
& \subseteq\left\{x^{*} \in \mathbb{R}^{k} \mid\left(x^{*},-v^{*}\right) \in \partial\left\langle y^{*}, g\right\rangle(\bar{x}, \bar{y}), y^{*} \in N_{D}(g(\bar{x}, \bar{y}))\right\} \\
& \subseteq\left\{x^{*} \in\left[\partial\left\langle y^{*}, g\right\rangle(\bar{x}, \bar{y})\right]_{x} \mid y^{*} \in N_{D}(g(\bar{x}, \bar{y}))\right\} .
\end{aligned}
$$

Let us write (3.8) in the form

$$
\left[\partial\left\langle y^{*}, g\right\rangle(\bar{x}, \bar{y})\right]_{x} \cap-\operatorname{bd} N_{C}(\bar{x}) \neq \emptyset, \quad y^{*} \in N_{D}(g(\bar{x}, \bar{y})) \Longrightarrow y^{*}=0 .
$$

By combining (3.9) and (3.10), one obtains that

$$
D^{*} S^{-1}(\bar{x}, \bar{y})(0) \cap-\operatorname{bd} N_{C}(\bar{x})=\{0\}
$$

and

$$
D^{*} S^{-1}(\bar{x}, \bar{y})\left(v^{*}\right) \cap-\operatorname{bd} N_{C}(\bar{x}) \neq \emptyset \Longrightarrow v^{*}=0 .
$$

These two conditions amount, however, to (3.1), and thus Theorem 3.2 can be applied to finish the proof.

The following example illustrates the application of Theorem 3.2 in the specific situation of Corollary 3.4.

Example 3.5. Define $M$ in (3.7) by $C=\left\{\left(x_{1}, x_{2}\right)\left|x_{2} \geq\right| x_{1} \mid\right\}, D=\mathbb{R}_{-}$, $g(x, y)=\min \left\{x_{1}, x_{2}\right\}-y$. Then, all data assumptions of Theorem 3.2 are satisfied at $\left(\bar{x}_{1}, \bar{x}_{2}, \bar{y}\right)=(0,0,0) \in \mathrm{Gph} M$, and also (3.1) holds true:

$$
\begin{gathered}
\bigcup_{y^{*} \in N_{D}(g(\bar{x}, \bar{y})) \backslash\{0\}}\left[\partial\left\langle y^{*}, g\right\rangle(\bar{x}, \bar{y})\right]_{x} \cap-\operatorname{bd} N_{C}(\bar{x}) \\
=\bigcup_{y^{*}>0} y^{*} \partial \min \{\cdot, \cdot\}(0,0) \cap \operatorname{bd} C \\
=\left\{\left(x_{1}, x_{2}\right) \mid x_{1}+x_{2}>0, x_{1} \cdot x_{2}=0\right\} \cap \operatorname{Gph}|\cdot|=\emptyset .
\end{gathered}
$$

Consequently, the calmness of $M$ in (3.7) can be derived. Note that the stronger criterion (1.6) ensuring the Aubin property of $M$ fails to apply here due to

$$
\left\{\left(x_{1}, x_{2}\right) \mid x_{1}+x_{2}>0, x_{1} \cdot x_{2}=0\right\} \cap-N_{C}(\bar{x})=\left\{\left(0, x_{2}\right) \mid x_{2}>0\right\} \neq \emptyset .
$$

At the same time, the contingent derivative criterion (1.2) for calmness on selections does not apply either, due to $M(0)=\left\{\left(x_{1}, x_{2}\right) \mid x_{2} \geq-x_{1} \geq 0\right\}$ not being singlevalued.

The following theorem provides a calmness result for the system (1.4) of functional constraints with canonical perturbations. In contrast to Theorem 3.2, no regularity or semismoothness assumption on $C$ will be made. Rather, the regularity assumption is shifted to the perturbed part of the constraints.

TheOREM 3.6. In (1.4) let $g$ be Lipschitz near $\bar{x} \in M(0)$, and $D$ be regular at $g(\bar{x})$. Further assume that the function $\left\langle y^{*}, g\right\rangle(\cdot)$ is regular at $\bar{x}$ for all $y^{*} \in \partial d_{D}^{e}(g(\bar{x}))$ and that the qualification condition

$$
\text { int } \bigcup_{y^{*} \in N_{D}(g(\bar{x})) \cap \mathbb{B}} \partial\left\langle y^{*}, g\right\rangle(\bar{x}) \cap-\left[T_{C}(\bar{x})\right]^{0} \neq \emptyset
$$


holds true. Then $M$ is calm at $(0, \bar{x})$.

Proof. Consider the composite function $\pi(x)=d_{D}^{e}(g(\bar{x}))$, which is evidently Lipschitz near $\bar{x}$ and for which one has $\pi(\bar{x})=0$. From [27, Theorem 10.49] we know that under our assumptions $\pi$ is even regular at $\bar{x}$ and

$$
\partial \pi(\bar{x})=\bigcup_{y^{*} \in N_{D}(g(\bar{x})) \cap \mathbb{B}} \partial\left\langle y^{*}, g\right\rangle(\bar{x}) .
$$

From (3.11) and (3.12) we infer the existence of some $\tilde{z}^{*} \in-\left[T_{C}(\bar{x})\right]^{0}$ and of some $\alpha>$ 0 such that $B\left(\tilde{z}^{*}, \alpha\right) \subseteq \partial \pi(\bar{x})$. Then, regularity of $\pi$ at $\bar{x}$ implies that $\left\langle\tilde{z}^{*}+\alpha p^{*}, h\right\rangle \leq$ $\pi^{\prime}(\bar{x} ; h)$ for all $p^{*} \in \mathbb{B}$ and all $h \in \mathbb{R}^{k}$, where $\pi^{\prime}(\bar{x} ; h)$ refers to the conventional directional derivative of $\pi$ taken at $\bar{x}$ in direction $h$. Consequently,

$$
\alpha\left\langle p^{*}, h\right\rangle \leq \pi^{\prime}(\bar{x} ; h)-\left\langle\tilde{z}^{*}, h\right\rangle \leq \pi^{\prime}(\bar{x} ; h) \quad \forall p^{*} \in \mathbb{B}, \forall h \in T_{C}(\bar{x}) .
$$

For arbitrary $h \in T_{C}(\bar{x}) \cap \mathbb{S}$ we set $p^{*}:=h$ and derive from the last relation that

$$
\pi^{\prime}(\bar{x} ; h) \geq \alpha>0 \quad \forall h \in T_{C}(\bar{x}) \cap \mathbb{S} .
$$

Assume that $M$ fails to be calm at $(0, \bar{x})$. Then, as in the proof of Theorem 3.2, there exist sequences $x_{n} \rightarrow \bar{x}, y_{n} \rightarrow 0, x_{n} \in M\left(y_{n}\right)$ such that $d\left(x_{n}, M(0)\right)>n\left\|y_{n}\right\|$. From here we deduce that $x_{n} \neq \bar{x}, x_{n} \in C$, and $\left\|x_{n}-\bar{x}\right\|>n\left(\pi\left(x_{n}\right)-\pi(\bar{x})\right)$ for all $n$. This amounts to $\left\|x_{n}-\bar{x}\right\|^{-1}\left(\pi\left(x_{n}\right)-\pi(\bar{x})\right)<n^{-1}$. It suffices now to pass to an appropriate subsequence $\left\{x_{n^{\prime}}\right\}$ such that $\left\|x_{n^{\prime}}-\bar{x}\right\|^{-1}\left(x_{n^{\prime}}-\bar{x}\right) \rightarrow h$ for some $h \in T_{C}(\bar{x}) \cap \mathbb{S}$. Local Lipschitz continuity of $\pi$ yields that $\pi^{\prime}(\bar{x} ; h)=0$, which contradicts $(3.13)$ and thus proves the calmness of $M$ at $(0, \bar{x})$.

REMARK 3.7. From (3.13) it immediately follows that (3.11) implies not only the calmness of $M$ at $(0, \bar{x})$ but also the isolatedness of $\bar{x}$ in $M(0)$, i.e., $\mathcal{U} \cap M(0)=\{\bar{x}\}$ for some neighborhood $\mathcal{U}$ of $\bar{x}$.

Example 3.5 shows that the last remark does not apply to the setting of Theorem 3.2 or Corollary 3.4 , where no regularity assumptions are made with respect to $S$ or $g$.

\section{Calmness in applications.}

4.1. Nonsmooth calculus. As shown, e.g., in [2], [4], [28], calmness plays an important role in deriving optimality conditions and in construction of local Lipschitz error bounds. It enables us, among other things, to replace the constraint system

$$
g(x) \in D, \quad x \in C,
$$

by a more easily tractable constraint

$$
(y, x) \in \operatorname{Gph} M,
$$

where $M$ is given by (3.7), and the new variable $y$ enters the objective via a suitable penalty term. Clearly, the feasible set given by (4.1) amounts to $M(0)$. For the evaluation of the normal cone to $M(0)$ at a given point $\bar{x}$, one usually employs various constraint qualifications. A prominent place is occupied by the MangasarianFromovitz constraint qualification, which in case of (4.1) becomes (1.6). Condition (1.6) ensures the Aubin property of $M$ around $(0, \bar{x})$ and, a fortiori, the inclusion

$$
N_{M(0)}(\bar{x}) \subset \bigcup_{y^{*} \in N_{D}(g(\bar{x}))} D^{*} g(\bar{x})\left(y^{*}\right)+N_{C}(\bar{x}) .
$$


It turns out, however, that the calmness of $M$ at $(0, \bar{x})$ also implies (4.2), and therefore, at least in some cases, condition (1.6) can be weakened.

THeOREM 4.1. Consider the multifunction $M$ given by (1.4) and a pair $(0, \bar{x}) \in$ Gph $M$. Assume that $g$ is Lipschitz near $\bar{x}$ and that $M$ is calm at $(0, \bar{x})$. Then inclusion (4.2) holds true.

Proof. We start with the observation that (see [22, Theorem 6.10])

$$
N_{\mathrm{Gph} M}(0, \bar{x}) \subset\left\{\left(y^{*}, x^{*}\right) \mid y^{*} \in N_{D}(g(\bar{x})), x^{*} \in \partial\left\langle y^{*}, g\right\rangle(\bar{x})+N_{C}(\bar{x})\right\} .
$$

Let $L$ be the modulus of calmness of $M$ at $(0, \bar{x})$. We claim that

$$
\forall x^{*} \in \partial d_{M(0)}^{e}(\bar{x}) \quad \exists y^{*} \in L \mathbb{B}:\left(y^{*}, x^{*}\right) \in N_{\mathrm{Gph} M}(0, \bar{x}) .
$$

To see this, note that $x^{*} \in \partial d_{M(0)}^{e}(\bar{x})$ means the existence of sequences $x_{n} \rightarrow \bar{x}$ $\left(x_{n} \in M(0)\right), r_{n} \downarrow 0, x_{n}^{*} \rightarrow x^{*}$, and $\varepsilon_{n} \downarrow 0$ such that

$$
d_{M(0)}^{e}(x)-d_{M(0)}^{e}(\bar{x}) \geq\left\langle x_{n}^{*}, x-x_{n}\right\rangle-\varepsilon_{n}\left\|x-x_{n}\right\| \quad \forall x \in B\left(x_{n}, r_{n}\right) .
$$

Since $M$ is calm at $(0, \bar{x})$, along with $L>0$ there exists some $r>0$ such that

$$
d_{M(0)}^{e}(x) \leq L\|y\| \quad \forall x \in B(\bar{x}, r) \cap M(y), \forall y \in B(0, r) .
$$

This implies that

$$
\begin{aligned}
& L\|y\|-\left\langle x_{n}^{*}, x-x_{n}\right\rangle+\varepsilon_{n}\left\|x-x_{n}\right\| \geq 0 \\
& \forall(y, x) \in \operatorname{Gph} M \cap\left(B(0, r) \times B\left(x_{n}, r_{n}\right)\right)
\end{aligned}
$$

for sufficiently large $n$. The function of $(y, x)$ on the left-hand side of (4.6) attains a constrained minimum at $\left(0, x_{n}\right)$. According to Proposition 4.3.4 in [4], the function

$$
L\|y\|-\left\langle x_{n}^{*}, x-x_{n}\right\rangle+\varepsilon_{n}\left\|x-x_{n}\right\|+K d_{\mathrm{Gph} M}^{e}(y, x)
$$

attains an unconstrained local minimum at $\left(0, x_{n}\right)$ for sufficiently large penalty parameter $K$. The respective optimality conditions imply the existence of some $y_{n}^{*} \in L \mathbb{B}$ such that

$$
0 \in\left\{-y_{n}^{*}\right\} \times\left(\left\{-x_{n}^{*}\right\}+\varepsilon_{n} \mathbb{B}\right)+N_{\mathrm{Gph} M}\left(0, x_{n}\right) .
$$

We now let $n$ tend to infinity and, passing to a subsequence $\left\{y_{n^{\prime}}^{*}\right\}$, establish the existence of a limit vector $y^{*} \in L \mathbb{B}$ such that $\left(y^{*}, x^{*}\right) \in N_{\mathrm{Gph} M}(0, \bar{x})$. This proves (4.4). It remains to observe that for each $\xi \in N_{M(0)}(\bar{x})$ there is some $x^{*} \in \partial d_{M(0)}^{e}(\bar{x})=$ $N_{M(0)}(\bar{x}) \cap \mathbb{B}$ such that $\xi=\|\xi\| x^{*}$. Since $g$ is Lipschitz, the result follows from (4.3) and (4.4).

Corollary 4.2. In (1.4), let $k=m$ and $\bar{x} \in C \cap D$. Assume that the map

$$
\tilde{M}(y):=\{x \in C \mid x+y \in D\}
$$

is calm at $(0, \bar{x})$. Then one has

$$
N_{C \cap D}(\bar{x}) \subset N_{C}(\bar{x})+N_{D}(\bar{x}) .
$$

Proof. It suffices to specialize the statement of Theorem 4.1 for $g$ being the identity mapping. 
REMARK 4.3. The calmness of $\tilde{M}$ at $(0, \bar{x})$ is closely related to the so-called metric inequality for the sets $C, D$ at $\bar{x}$ [12], which also implies inclusion (4.7).

In the literature (e.g., [20], [22]) one usually requires the qualification condition

$$
N_{D}(\bar{x}) \cap-N_{C}(\bar{x})=\{0\}
$$

to ensure the validity of inclusion (4.7). However, condition (4.8) implies the Aubin property of $\tilde{M}$ around $(0, \bar{x})$ and is thus clearly more demanding than the calmness required in Corollary 4.2.

By combining Theorem 3.2 and the above corollary, we immediately conclude that, to ensure inclusion (4.7), it suffices to replace (4.8) by a weaker condition

$$
N_{D}(\bar{x}) \cap-\operatorname{bd} N_{C}(\bar{x})=\{0\}
$$

whenever $C$ is regular and semismooth at $\bar{x}$. Moreover, as observed by Kruger [14], condition (4.9) alone (without regularity or semismoothness assumptions) implies inclusion (4.7). The respective statement can be formulated even for a general mapping $M$ permitting noncanonical perturbations.

Proposition 4.4 (adapted from [14]). Consider the map $M$ given by (3.7), where $g$ is Lipschitz around a reference pair $(\bar{y}, \bar{x}) \in \mathrm{Gph} M$ and $C, D$ are closed subsets of the respective spaces. Assume that (3.8) is fulfilled. Then either $M$ possesses the Aubin property around $(\bar{y}, \bar{x})$ or

$$
\bigcup_{y^{*} \in N_{D}(g(\bar{x}, \bar{y})) \backslash\{0\}}\left[\partial\left\langle y^{*}, g\right\rangle(\bar{x}, \bar{y})\right]_{x}+N_{C}(\bar{x})=\mathbb{R}^{p} .
$$

Proof. If

$$
\bigcup_{y^{*} \in N_{D}(g(\bar{x}, \bar{y})) \backslash\{0\}}\left[\partial\left\langle y^{*}, g\right\rangle(\bar{x}, \bar{y})\right]_{x} \cap-N_{C}(\bar{x})=\emptyset,
$$

then it follows from [22, Theorem 6.10] that

$$
\begin{gathered}
D^{*} M(\bar{y}, \bar{x})\left(x^{*}\right) \subset\left\{y^{*} \in \mathbb{R}^{m} \mid\left(y^{*},-x^{*}\right) \in D^{*} g(\bar{x}, \bar{y}) \circ N_{D}(g(\bar{x}, \bar{y}))\right. \\
\left.+\left(0 \times N_{C}(\bar{x})\right)\right\} .
\end{gathered}
$$

Combining (4.11) and (4.12) provides $D^{*} M(\bar{y}, \bar{x})(0)=\{0\}$, whence the Aubin property of $M$ at $(\bar{y}, \bar{x})$ (see (1.1)). According to (3.8), assume therefore that

$$
\bigcup_{y^{*} \in N_{D}(g(\bar{x}, \bar{y})) \backslash\{0\}}\left[\partial\left\langle y^{*}, g\right\rangle(\bar{x}, \bar{y})\right]_{x} \cap-\operatorname{int} N_{C}(\bar{x}) \neq \emptyset .
$$

Then

$$
\exists y^{*} \in N_{D}(g(\bar{x}, \bar{y})) \backslash\{0\}, \exists x^{*} \in\left[\partial\left\langle y^{*}, g\right\rangle(\bar{x}, \bar{y})\right]_{x}, \exists \alpha>0: \quad B\left(x^{*}, \alpha\right) \subset-N_{C}(\bar{x}) .
$$

This implies for each $p^{*} \in B(0, \alpha)$ that

$$
p^{*} \in \bigcup_{y^{*} \in N_{D}(g(\bar{x}, \bar{y})) \backslash\{0\}}\left[\partial\left\langle y^{*}, g\right\rangle(\bar{x}, \bar{y})\right]_{x}+N_{C}(\bar{x}) .
$$

Now, the result follows. 
Corollary 4.5. Let $C, D \subseteq \mathbb{R}^{k}$ be arbitrary closed sets with $\bar{x} \in C \cap D$. Then (4.9) ensures inclusion (4.7).

Proof. Apply Proposition 4.4 with $g(x, y):=x$.

According to the proof of Proposition 4.4, the difference between (3.8) and the classical Mangasarian-Fromovitz constraint qualification (4.11) reduces to the case (4.13), for which the argument from Remark 3.7 implies the local isolatedness of the feasible points of $M(0)$ (under the additional assumptions of Theorem 3.6). This fact is easily interpreted for mathematical programs of the form

$$
\min \{f(x) \mid x \in M(0)\} .
$$

Evidently, isolated points of $M(0)$ are automatically local minima; hence, in this context (3.8) goes beyond the Mangasarian-Fromovitz constraint qualification as a condition providing nondegenerate Lagrange multipliers, in that it identifies local minima given by isolated feasible points.

Another observation is the following: Since polyhedral mappings are automatically calm (cf. [26]), we derive from Theorem 4.1 that a nonsmooth calculus rule like (4.2) can be obtained under no constraint qualifications for polyhedral data.

4.2. First-order growth (weak sharp minima), local uniqueness, and stability of solutions. Consider the problem

$$
\text { (P) } \min \{f(x) \mid x \in C\},
$$

where $f: \mathbb{R}^{k} \rightarrow R$ is a continuous function and $C \subseteq \mathbb{R}^{k}$ a closed subset. Denote the solution set of $(P)$ by $S$. Recall the following definition.

DeFINITION 4.6. In $(P)$, the objective function $f$ is said to satisfy a first-order growth condition if there exist a constant $c>0$ and a neighborhood $\mathcal{N}$ of $S$ such that

$$
f(x) \geq f_{*}+c d(x, S) \quad \forall x \in C \cap \mathcal{N},
$$

where $f_{*}=\inf \{f(x) \mid x \in C\}$. Equivalently, $f$ is said to have a set $S$ of weak sharp minima with respect to $C \cap \mathcal{N}$ (cf. [3]).

LEMma 4.7. Let the solution set $S$ of $(P)$ be nonempty and bounded, and suppose that the multifunction $M(y):=\{x \in C \mid f(x) \leq y\}$ is calm on $\left\{f_{*}\right\} \times S$ (i.e., calm at all $\left(f_{*}, x\right)$ with $\left.x \in S\right)$. Then, $f$ satisfies a first-order growth condition in $(P)$.

Proof. Fix an arbitrary $x^{0} \in S$. Obviously, $f\left(x^{0}\right)=f_{*}$; hence the calmness of $M$ at $\left(f\left(x^{0}\right), x^{0}\right)$ implies the existence of $\varepsilon, \delta, L>0$ such that

$$
d\left(x, M\left(f\left(x^{0}\right)\right)\right) \leq L\left|y-f\left(x^{0}\right)\right| \quad \forall y:\left|y-f\left(x^{0}\right)\right|<\delta, \forall x \in M(y) \cap B\left(x^{0}, \varepsilon\right) .
$$

Choose $\varepsilon>0$ small enough to meet $\left|f(x)-f\left(x^{0}\right)\right|<\delta$ for all $x \in B\left(x^{0}, \varepsilon\right)$. Now, one may put $y:=f(x)$ in the above estimation and derive from $M\left(f\left(x^{0}\right)\right)=S$ that

$$
d(x, S) \leq L\left|f(x)-f\left(x^{0}\right)\right| \quad \forall x \in C \cap B\left(x^{0}, \varepsilon\right) .
$$

From $f(x) \geq f\left(x^{0}\right)$ for all $x \in C$, it follows that

$$
f(x) \geq f_{*}+L^{-1} d(x, S) \quad \forall x \in C \cap B\left(x^{0}, \varepsilon\right) .
$$

By our assumptions, $S$ is compact. Hence, a finite number of $x^{i} \in S, \varepsilon_{i}>0$, and $L_{i}>0$ exists such that $S \subseteq \cup_{i} B\left(x^{i}, \varepsilon_{i}\right)$ and

$$
f(x) \geq f_{*}+L_{i}^{-1} d(x, S) \quad \forall x \in C \cap B\left(x^{\forall i}, \varepsilon_{i}\right) .
$$


This, however, implies that $f$ satisfies a first-order growth condition with $c:=\left(\max L_{i}\right)^{-1}$ and $\mathcal{N}:=\cup_{i} B\left(x^{i}, \varepsilon_{i}\right)$.

Corollary 4.8. In $(P)$ let $f$ be locally Lipschitz and $C$ be regular and semismooth. Then $f$ satisfies a first-order growth condition if the solution set $S$ is nonempty and bounded and, moreover, the condition

$$
\partial f(x) \cap-\operatorname{bd} N_{C}(x)=\emptyset \quad \forall x \in S
$$

holds true.

Proof. Combine Lemma 4.7 with Corollary 3.4 (setting $g(x, y):=f(x)+y$ and $D:=\mathbb{R}_{\text {- there) } \text {. }}$

A consequence of the constraint qualification in the last corollary is that solutions are locally isolated, as described in the following.

Proposition 4.9. Let $f: \mathbb{R}^{k} \rightarrow \mathbb{R}$ be Lipschitz near $\bar{x} \in S$, and $C \subseteq \mathbb{R}^{k}$ be regular at $\bar{x}$. If, in addition, $C$ or $f$ is semismooth at $\bar{x}$, then the condition $\partial f(\bar{x}) \cap$ $-\operatorname{bd} N_{C}(\bar{x})=\emptyset$ entails that $\mathcal{U} \cap S=\{\bar{x}\}$ for some neighborhood $\mathcal{U}$ of $\bar{x}$.

Proof. Assume, by contradiction, that $x_{n} \rightarrow \bar{x}$ for some sequence $x_{n} \in S \backslash\{\bar{x}\}$. Then, without loss of generality, $\left\|x_{n}-\bar{x}\right\|^{-1}\left(x_{n}-\bar{x}\right) \rightarrow h \in T_{C}(\bar{x})$. On the other hand, as $x_{n} \in S$, it follows that $f\left(x_{n}\right)=f(\bar{x})$ and $0 \in \partial f\left(x_{n}\right)+N_{C}\left(x_{n}\right)$. Accordingly, we may extract a sequence $y_{n}^{*} \in \partial f\left(x_{n}\right) \cap-N_{C}\left(x_{n}\right)$. This sequence is bounded because $f$ is Lipschitz around $\bar{x}$. Hence, without loss of generality, $y_{n}^{*} \rightarrow y^{*}$ for some $y^{*} \in \partial f(\bar{x}) \cap-N_{C}(\bar{x})$. We claim that $y^{*} \in-\operatorname{bd} N_{C}(\bar{x})$, whence a contradiction to the assumed condition $\partial f(\bar{x}) \cap-\operatorname{bd} N_{C}(\bar{x})=\emptyset$. Indeed, if $C$ is semismooth at $\bar{x}$, this is an immediate consequence of Proposition 3.1. In the opposite case, the semismoothness of $f$ at $\bar{x}$ provides that

$$
\left\langle y_{n}^{*}, h\right\rangle \rightarrow\left\langle y^{*}, h\right\rangle=f^{\prime}(\bar{x} ; h)=\lim _{n \rightarrow \infty}\left\|x_{n}-\bar{x}\right\|^{-1}\left(f\left(x_{n}\right)-f(\bar{x})\right)=0 .
$$

Now the same reasoning as in the proof of Proposition 3.1 allows us to derive that $y^{*} \in-\operatorname{bd} N_{C}(\bar{x})$.

Evidently, Proposition 4.9 may be taken as a subdifferential condition for the local uniqueness of solutions. Now we are in a position to state a subdifferential condition for upper Lipschitz stability of solution sets. Consider the parametric optimization problem

$$
P(y) \quad \min \{f(x) \mid g(x) \leq y\},
$$

where $f: \mathbb{R}^{k} \rightarrow \mathbb{R}$ and $g: \mathbb{R}^{k} \rightarrow \mathbb{R}^{m}$ are locally Lipschitz, and $M(y)$ and $S(y)$ denote the parameter-dependent sets of feasible points and solutions, respectively. The set of active indices at $x$ in the relation $g(x) \leq y$ will be denoted by $I(x)$.

THEOREM 4.10. Let $S(0)$ be nonempty and bounded, and assume the following conditions to hold true for all $x \in S(0)$ :

(1) All components $g_{i}$ of $g$ are regular and semismooth at $x$.

(2) $\partial f(x) \cap-\operatorname{bd} N_{g^{-1}\left(\mathbb{R}_{-}^{m}\right)}(x)=\emptyset$.

(3) $0 \notin$ bd conv $\left.\left\{\partial g_{i}(x)\right\} \mid i \in I(x)\right\}$ ("conv"= convex hull).

Then, there exist some neighborhood $\mathcal{U}$ of $S(0)$ and constants $\varepsilon, L>0$ such that

$$
d(x, S(0)) \leq L\|y\| \quad \forall y \in B(0, \varepsilon), \forall x \in \mathcal{U} \cap S(y) .
$$

Proof. We shall show that $S$ is calm at $(0, x)$ for all $x \in S(0)$ and that $S(0)$ consists just of isolated points. Given this fact, our compactness assumption ensures 
that $S(0)$ will consist of only finitely many points, say $S(0)=\left\{x^{1}, \ldots, x^{N}\right\}$. The calmness property then means the existence of constants $L_{i}, \varepsilon_{i}, \delta_{i}$ such that

$$
d(x, S(0)) \leq L_{i}\|y\| \quad \forall y \in B\left(0, \varepsilon_{i}\right), \forall x \in B\left(x^{i}, \delta_{i}\right) \cap S(y)(i=1, \ldots, N) .
$$

Setting $L:=\max L_{i}, \varepsilon:=\min \varepsilon_{i}$, and $\mathcal{U}:=\cup B\left(x^{i}, \delta_{i}\right)$, the assertion of the theorem follows.

In order to prove the stated facts, let $\bar{x} \in S(0)$ be arbitrarily given. Note that our constraint system $M(y)=\{x \mid g(x) \leq y\}$ is a special case of (1.4) with $D:=\mathbb{R}_{-}^{m}$ and $C:=\mathbb{R}^{k}$. It is easily checked that assumption (1) implies the setting considered in Theorem 3.6. Indeed, regularity of the $g_{i}$ implies regularity of any function $\sum_{i=1}^{m} y_{i}^{*} g_{i}$ with $y_{i}^{*} \geq 0$; hence $\left\langle y^{*}, g\right\rangle$ is regular at $\bar{x}$ for all

$$
y^{*} \in \partial d_{D}^{e}(g(\bar{x}))=N_{D}(g(\bar{x})) \cap \mathbb{B}=\left\{y^{*} \in \mathbb{R}_{+}^{m} \mid\left\|y^{*}\right\| \leq 1, y_{i}^{*}=0(i \notin I(\bar{x}))\right\},
$$

as required in Theorem 3.6.

Suppose first that $0 \in \operatorname{int} H$, where $\left.H:=\operatorname{conv}\left\{\partial g_{i}(\bar{x})\right\} \mid i \in I(\bar{x})\right\}$. By regularity of the $g_{i}$, the subdifferentials $\partial g_{i}(\bar{x})$ are convex; hence

$$
H=\left\{\sum_{i \in I(\bar{x})} y_{i}^{*} \partial g_{i}(\bar{x}) \mid \sum_{i \in I(\bar{x})} y_{i}^{*}=1, y_{i}^{*} \geq 0\right\} .
$$

Therefore

$$
H \subseteq \bigcup_{y^{*} \in N_{D}(g(\bar{x})) \cap \mathbb{B}} \partial\left\langle y^{*}, g\right\rangle(\bar{x}),
$$

which along with $\left[T_{C}(\bar{x})\right]^{0}=\{0\}$ implies that (3.11) holds. Hence, by Remark 3.7, $M(0)$ is locally isolated at $\bar{x}$. Then, $S(0)$ is isolated at $\bar{x}$ as well due to $S(0) \subseteq M(0)$. Furthermore, Theorem 3.6 allows us to derive the calmness of $M$ at $(0, \bar{x})$, i.e.,

$$
d(x, M(0)) \leq L\|y\| \quad \forall y \in B(0, \varepsilon), \forall x \in \mathcal{V} \cap M(y)
$$

for some neighborhood $\mathcal{V}$ of $\bar{x}$ and some $\varepsilon, L>0$. Choosing $\mathcal{V}$ small enough to meet $d(x, S(0))=\|x-\bar{x}\|$ (by the local isolatedness of $S(0)$ ), one may conclude that

$$
d(x, S(0)) \leq d(x, M(0)) \leq L\|y\| \quad \forall y \in B(0, \varepsilon), \forall x \in \mathcal{V} \cap S(y),
$$

where we used once more that $S(y) \subseteq M(y)$. This, however, is calmness of $S$ at $\bar{x}$.

In the opposite case, $0 \notin \operatorname{int} H$, assumption (3) entails that $0 \notin H$. This condition along with assumption (1) implies the regularity and semismoothness of the set $g^{-1}\left(\mathbb{R}_{-}^{m}\right)$ at $\bar{x}$ (see [8, Lemma 3.6]). Then, in view of our assumptions, Proposition 4.9 may be invoked to show the local isolatedness of $S(0)$ at $\bar{x}$ again. Furthermore, the condition $0 \notin H$ is nothing but the Mangasarian-Fromovitz constraint qualification for a finite set of locally Lipschitz inequalities. It is well known that then the constraint mapping $M$ has even the Aubin property around $(0, \bar{x})$, which is stronger than calmness. Hence, exactly the same argument as in the previous case can be applied to derive the calmness of $S$ at $(0, \bar{x})$.

Concerning the first assumption in Theorem 4.10, an analogous statement to that of Remark 3.3 applies. In particular, convex and $\mathcal{C}^{1}$-functions are regular and semismooth (even a maximum of such functions). 
The next example illustrates the application of Theorem 4.10 in a smooth setting and, along the way, demonstrates how the upper Lipschitz stability of solutions can be established despite violation of the Mangasarian-Fromovitz constraint qualification.

EXAMPLE 4.11. Consider the parametric optimization problem

$$
\min \left\{\left(x_{1}-1 / 2\right)^{2} \mid-x_{1}-x_{2} \leq y_{1} ; x_{2} \leq y_{2} ; x_{1}\left(1-x_{1}\right)-x_{2} \leq y_{3}\right\} .
$$

Then $S(0)=\left\{x^{a}, x^{b}\right\}$ with $x^{a}=(0,0), x^{b}=(1,0)$. Obviously, $S(0)$ is nonempty and bounded, and the constraint functions satisfy assumption (1) of Theorem 4.10 by smoothness. At $x^{a}$ all unperturbed constraints are binding; hence the set $H$ from assumption (3) is given as the convex hull of the three gradients:

$$
H=\operatorname{conv}\{(-1,-1),(0,1),(1,-1)\} .
$$

Obviously, $0 \in \operatorname{int} H$; hence the Mangasarian-Fromovitz constraint qualification is violated at $x^{a}$. In contrast, the condition $0 \notin$ bd $H$ of assumption (3) is fulfilled. Furthermore, $0 \in \operatorname{int} H$ implies that the unperturbed constraint set $M(0)=g^{-1}\left(\mathbb{R}_{-}^{3}\right)$ is locally isolated at $x^{a}$ (see the proof of Theorem 4.10). Therefore, $N_{g^{-1}\left(\mathbb{R}_{-}^{3}\right)}\left(x^{a}\right)=\mathbb{R}^{2}$, and assumption (2) holds trivially. Concerning $x^{b}$, only the second and third constraint are binding, so $H=\operatorname{conv}\{(0,1),(-1,-1)\}$ and $0 \notin H$. Again, assumption (3) is satisfied. Moreover, $N_{g^{-1}\left(\mathbb{R}^{3}\right)}\left(x^{b}\right)$ is the convex cone generated by the two active gradients $(0,1)$ and $(-1,-1)$, so its negative boundary is $\left(\mathbb{R}_{+} \cdot(0,-1)\right) \cup\left(\mathbb{R}_{+} \cdot(1,1)\right)$. Again, assumption (2) is fulfilled. Summarizing, the upper Lipschitz behavior of solutions to the above parametric problem can be derived.

4.3. Equilibrium mappings. In [23] and [6] the authors study various stability properties of parametrized equilibria governed by the generalized equations

$$
0 \in f(x, y)+Q(x)
$$

where $x \in \mathbb{R}^{k}$ is the decision variable, $y \in \mathbb{R}^{p}$ is the parameter, $f: \mathbb{R}^{k} \times \mathbb{R}^{p} \rightarrow \mathbb{R}^{k}$ is continuously differentiable, and $Q: \mathbb{R}^{k} \rightrightarrows \mathbb{R}^{k}$ is a closed-valued multifunction. If one considers an optimization problem with (4.15) as a constraint, and an additional abstract constraint $(x, y) \in C$, then it is important to verify the calmness of the mapping $H: \mathbb{R}^{k} \rightrightarrows \mathbb{R}^{k} \times \mathbb{R}^{p}$ defined by

$$
H(z):=\{(x, y) \in C \mid z \in f(x, y)+Q(x)\} .
$$

$H$ can easily be converted to the form (3.7), and so Corollary 3.4 can be applied. In fact, this procedure is illustrated in [8] by a parameterized equilibrium governed by a nonlinear complementarity problem. In this section we concentrate on a different mapping associated with parameterized equilibria, namely, the intersection

$$
\Theta(y):=S(y) \cap C,
$$

where $S$ is the so-called solution mapping defined by

$$
S(y)=\left\{x \in \mathbb{R}^{k} \mid 0 \in f(x, y)+Q(x, y)\right\},
$$

and $C$ is a closed subset of $\mathbb{R}^{k}$ specifying the feasible decision variables. In (4.16) we admit that $Q$ also depends on the parameter $y$, which extends the class of considered equilibria. Calmness of $S$ (with $Q$ depending only on $x$ ) has been investigated in [6], 
but in the narrower sense of calmness on selections (see the introduction) where, for a reference pair $(\bar{y}, \bar{x})$, one requires $\bar{x}$ to be an isolated point of $S(\bar{y})$.

The mapping $S$ can be written in the form $S(y)=\left\{x \in \mathbb{R}^{k} \mid g(x, y) \in D\right\}$, where $g(x, y)=(x, y,-f(x, y))^{T}$ and $D=\operatorname{Gph} Q$. Therefore, $\Theta$ has exactly the structure of the multifunction $M$ in (3.7), and we immediately obtain the following statement from Corollary 3.4.

THEOREM 4.12. Let $C$ be regular and semismooth at $\bar{x} \in \Theta(\bar{y})$. Further assume that the qualification condition

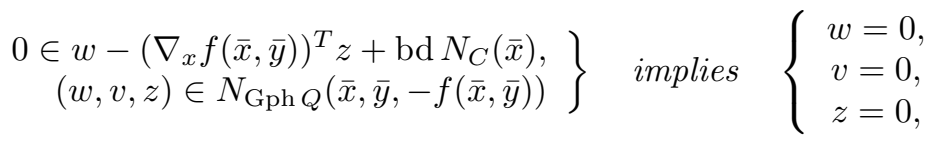

holds true. Then $\Theta$ is calm at $(\bar{y}, \bar{x})$.

If $Q$ depends just on $x$, then $g(x, y)=(x,-f(x, y))^{T}$, and the qualification condition (4.17) reduces to

$$
\left.\begin{array}{r}
0 \in w-\left(\nabla_{x} f(\bar{x}, \bar{y})\right)^{T} z+\operatorname{bd} N_{C}(\bar{x}), \\
(w, z) \in N_{\operatorname{Gph} Q}(\bar{x},-f(\bar{x}, \bar{y}))
\end{array}\right\} \quad \text { implies } \quad\left\{\begin{array}{l}
w=0 \\
z=0 .
\end{array}\right.
$$

The following example shows that the qualification conditions (4.17), (4.18) may well be violated even when $\Theta$ is calm at $(\bar{y}, \bar{x})$.

EXAMPLE 4.13. In (4.16) let $k=p=1, f \equiv 0$, and

$$
Q(x, y)=\partial \varphi(x)+N_{y+\mathbb{R}_{-}}(x), \quad \varphi(x)=\left\{\begin{array}{ccc}
-x & \text { for } & x \leq 0 \\
0 & \text { for } & x>0 .
\end{array}\right.
$$

Clearly,

$$
S(y)= \begin{cases}y & \text { for } y \leq 0 \\ {[0, y]} & \text { otherwise }\end{cases}
$$

Let $(\bar{y}, \bar{x})=(0,0)$. It is easily seen that with $C=\mathbb{R}_{+}$or $C=\mathbb{R}_{-}$the mapping $\Theta$ is calm at $(\bar{y}, \bar{x})$. Nevertheless, condition (4.17) is not fulfilled.

The reason for the failure of (4.17) in the last example is that this condition works with a too large upper approximation of $D^{*} S(\bar{y}, \bar{x})$. In such cases it makes sense to directly apply Theorem 3.2: In Example 4.13 one calculates

$$
D^{*} S^{-1}(\bar{y}, \bar{x})\left(y^{*}\right)=\left\{\begin{array}{lll}
y^{*} & \text { if } & y^{*} \neq 0 \\
\mathbb{R}_{-} & \text {if } & y^{*}=0 .
\end{array}\right.
$$

Both for $C=\mathbb{R}_{+}$and $C=\mathbb{R}_{-}$, it is easily verified that (3.1) holds true, and hence, calmness of $\Theta$ can be derived. Observe that this result could not be obtained when considering the whole cone $N_{C}(\bar{x})$ instead of its boundary.

REMARK 4.14. The calmness of $\Theta$ in the above example follows directly from its polyhedral nature. Nevertheless, it illustrates well the need to weaken the standard criteria ensuring the Aubin property when analyzing calmness.

Acknowledgments. The authors gratefully acknowledge support by the Weierstrass Institute Berlin, where this paper was prepared during the second and third authors' stay as visiting guests. They are also indebted to A. Kruger (Minsk) for helpful discussions on the subject of the paper, and to both referees for their valuable comments and suggestions. 


\section{REFERENCES}

[1] J.-P. Aubin And I. Ekeland, Applied Nonlinear Analysis, Wiley, New York, 1984.

[2] J.F. Bonnans And A. Shapiro, Perturbation Analysis of Optimization Problems, SpringerVerlag, Berlin, 2000.

[3] J.V. Burke AND M.C. FERRIS, Weak sharp minima in mathematical programming, SIAM J. Control Optim., 31 (1993), pp. 1340-1359.

[4] F.H. Clarke, Nonsmooth Analysis and Optimization, Wiley, New York, 1983.

[5] S. DENG, Global error bounds for convex inequality systems in Banach spaces, SIAM J. Control. Optim., 36 (1998), pp. 1240-1249.

[6] A.L. Dontchev and R.T. Rockafellar, Ample Parameterization of Variational Inclusions, SIAM J. Optim., 12 (2001), 170-187.

[7] R. Henrion, The Approximate Subdifferential and Parametric Optimization, Habilitation thesis, Humboldt University, Berlin, Germany, 1997.

[8] R. Henrion And J. Outrata, A subdifferential condition for calmness of multifunctions, J. Math. Anal. Appl., 258 (2001), pp. 110-130.

[9] R. Henrion And A. Jourani, Subdifferential systems for calmness for convex systems, SIAM J. Optim., 13 (2002), pp. 520-534.

[10] A.D. Ioffe, Necessary and sufficient conditions for a local minimum, 1: A reduction theorem and first order conditions, SIAM J. Control Optim., 17 (1979), pp. 245-250.

[11] A.D. Ioffe, Regular points of Lipschitz functions, Trans. Amer. Math. Soc., 251 (1979), pp. 61-69.

[12] A.D. Ioffe, Approximate subdifferential and applications, Part 3, Mathematika, 36 (1989), pp. 1-38.

[13] A.J. KIng AND R.T. RockAFELlar, Sensitivity analysis for nonsmooth generalized equations, Math. Programming, 55 (1992), pp. 193-212.

[14] A. KRUGER, private communication.

[15] A.B. LEvy, Implicit multifunction theorems for the sensitivity analysis of variational conditions, Math. Programming, 74 (1996), pp. 333-350.

[16] A.B. LEvY, Calm minima in parameterized finite-dimensional optimization, SIAM J. Optim., 11 (2000), pp. 160-178.

[17] A.B. LevY, Solution sensitivity from general principles, SIAM J. Control Optim., 40 (2001), pp. $1-38$.

[18] W. Li AND I. Singer, Global error bounds for convex multifunctions and applications, Math. Oper. Res., 23 (1998), pp. 443-462.

[19] R. Mifflin, Semismooth and semiconvex functions in constrained optimization, SIAM J. Control Optim., 15 (1977), pp. 959-972.

[20] B.S. Mordukhovich, Approximation Methods in Problems of Optimization and Control, Nauka, Moscow, 1988 (in Russian: Wiley-Interscience English translation to appear).

[21] B.S. Mordukhovich, Complete characterization of openness, metric regularity and Lipschitz properties of multifunctions, Trans. Amer. Math. Soc., 340 (1993), pp. 1-35.

[22] B.S. Mordukhovich, Generalized differential calculus for nonsmooth and set-valued mappings, J. Math. Anal. Appl., 183 (1994), pp. 250-288.

[23] B.S. Mordukhovich, Stability theory for parametric generalized equations and variational inequalities via nonsmooth analysis, Trans. Amer. Math. Soc., 343 (1994), pp. 609-655.

[24] J.-S. PANG, Error bounds in mathematical programming, Math. Programming, 79 (1997), pp. 299-332.

[25] S.M. Robinson, Generalized equations and their solutions, Part I: Basic theory, Math. Programming Study, 10 (1979), pp. 128-141.

[26] S.M. Robinson, Some continuity properties of polyhedral multifunctions, Math. Programming Study, 14 (1981), pp. 206-214.

[27] R.T. Rockafellar and R. Wets, Variational Analysis, Springer-Verlag, New York, 1999.

[28] J.J. YE AND X.Y. YE, Necessary optimality conditions for optimization problems with variational inequality constraints, Math. Oper. Res., 22 (1997), pp. 977-997. 